\title{
Principios de diseño de insignias digitales en programas de educación continua profesional: una revisión exploratoria
}

\author{
Ismael Canales-Negrón* \\ Pontificia Universidad Católica del Perú, Lima, Perú \\ https://orcid.org/0000-0002-2473-4602
}

Recibido:18/05/20 Revisado:31/07/20 Aceptado: 18/09/20 Publicado:3/11/20

\begin{abstract}
Resumen
Las insignias digitales son representaciones visuales de una competencia, habilidad o conocimiento adquirido por una persona. Su utilidad práctica es otorgar visibilidad a estas características mediante su difusión en plataformas digitales, además de almacenar datos y evidencias acerca del logro alcanzado. Esta nueva tecnología puede transformar los procesos de acreditación y evaluación en contextos educativos y corporativos, al proveer de información específica sobre el desempeño de los participantes en distintas actividades. Se realizó una revisión exploratoria de dieciséis proyectos de insignias digitales aplicados en programas de educación continua profesional. Los detalles sobre las tendencias de diseño y resultados de estos proyectos fueron publicados en veinte documentos registrados en las bases de datos Scopus y Web of Science. Se concluyó que los principios de diseño más utilizados fueron aquellos relacionados con el reconocimiento y evaluación de competencias, hecho que concuerda con el interés de los participantes en que los conocimientos aprendidos obtengan mayor visibilidad y utilidad para su desarrollo profesional. Se recomienda difundir los beneficios de las insignias digitales para enriquecer la experiencia de aprendizaje en programas educativos similares.
\end{abstract}

Palabras clave: Insignias digitales; Principios de diseño de insignias, Microcredenciales; Educación continua profesional

\section{Using digital badges design principles in professional continuing education programs: a scoping review}

\begin{abstract}
Digital badges are visual representations of a skill, ability or knowledge acquired by a person. Their practical usefulness is to grant these features visibility through broadcasting on online platforms, in addition to storing and publishing evidence and data about the depicted achievement. This new technology is capable of transforming accreditation and evaluation processes for educational and corporate contexts, by providing specific information participants performance in training or work related activities. An exploratory review of sixteen digital badge projects applied in continuing professional education programs was carried out. Details on the design trends and results of these projects were published in twenty documents registered in Scopus and Web of Science. It was determined that the most used design principles were those related to the recognition and evaluation of competences and skills, a situation that agrees with the interest of the participants in that the knowledge learned obtain greater visibility and usefulness for their professional development. In any case, it is recommended to offer more information about the benefits of digital badges to enrich and promote learning experiences in similar educational programs.
\end{abstract}

Keywords: Digital badges; Badges design principles, Microcredentials; Continuing professional education 


\title{
Principios de design de distintivos digitais para programas de educação profissional continuada: uma revisão exploratoria
}

\begin{abstract}
Resumo
Os emblemas digitais são representações visuais de competências, habilidades ou conhecimentos adquiridos por uma pessoa, cuja utilidade é dar visibilidade a essas características em um ambiente digital. Essa nova tecnologia pode transformar os processos de acreditação e avaliação, ao fornecer informações específicas sobre o desempenho dos participantes em treinamentos ou atividades de trabalho.

Foi realizada uma revisão exploratória de dezesseis projetos de emblemas digitais aplicados em programas de educação profissional continuada. Informações detalhadas sobre esses projetos foram publicadas em vinte documentos registrados no Scopus e Web of Science. Determinou-se que os princípios de design mais utilizados foram aqueles relacionados com o reconhecimento e avaliação de competências, situação que vai ao encontro do interesse dos participantes em que os conhecimentos apreendidos obtenham maior visibilidade e utilidade para o seu desenvolvimento profissional. Recomendase divulgar os benefícios dos emblemas digitais para enriquecer a experiência de aprendizagem em programas educacionais. Palavras-chave: Emblemas digitais; Princípios de design de emblemas digitais; Micro credenciais; Educação Profissional Continuada
\end{abstract}

Citar como:

Canales-Negrón, I. (2020). Principios de diseño de insignias digitales en programas de educación continua profesional: una revisión exploratoria. Revista Digital de Investigación en Docencia Universitaria, 14(2), e1170. https://doi. org/10.19083/ridu.2020.1170

\section{Introducción}

Uno de los factores de éxito de las organizaciones y empresas es disponer de un equipo de trabajadores que realicen un desempeño aceptable durante el desarrollo de las labores asignadas, para que alcancen altos niveles de productividad. Para mantener a sus trabajadores motivados y comprometidos con la institución, las empresas pueden tomar una serie de medidas, tales como promover el trabajo en equipo, construir relaciones sólidas con el empleador, ofrecer recompensas e incentivos en reconocimiento al buen desempeño y facilitar el crecimiento profesional de los trabajadores (Madero, 2009).

El escenario ideal para cualquier organización es que los profesionales tomen conciencia sobre su propio avance educativo y participen de manera voluntaria en diversos programas y cursos de actualización durante el tiempo que dure su actividad laboral. La idea de que las personas se involucren proactivamente en actividades formativas duraderas y a largo plazo, forma parte del aprendizaje permanente o lifelong learning. Este proceso de aprendizaje consiste en enriquecer los conocimientos aprendidos por una persona a lo largo de su vida, mediante la participación en actividades educativas destinadas a mejorar no solo su desempeño laboral sino también sus relaciones familiares y sociales, como parte de una continua búsqueda de crecimiento y desarrollo individual. (Curran et al., 2019; Taylor y Neymeyer, 2017)

Los programas de Educación Continua Profesional (ECP) forman parte del proceso de aprendizaje permanente, puesto que ofrecen un conjunto de cursos y servicios cuyo objetivo es actualizar los conocimientos adquiridos por los trabajadores durante sus estudios profesionales, además de aportar valores, conocimientos y habilidades focalizados en mejoras estrictamente laborales. Por lo general, estas actividades se realizan durante toda la carrera laboral y sirven de ayuda a 
los trabajadores para permanecer al tanto de las actualizaciones y nuevas tendencias dentro de su profesión (Livneh y Livneh, 1999; Pineda y Sarramona, 2006; Reynoso Flores et al., 2014).

Finalmente, la decisión de promover la formación profesional de los trabajadores dentro del centro de trabajo es el primer paso hacia la creación de una cultura de aprendizaje que apunta al desarrollo personal del trabajador, gracias a la adquisición de nuevos conocimientos que enriquecerán su experiencia laboral. La empresa también se ve beneficiada dentro de este contexto, pues estos espacios de aprendizaje mejorarán el clima organizacional al fortalecer la confianza, el compromiso y la colaboración entre todos sus miembros (Gil López y Gallego Gil, 2016).

Tradicionalmente el registro de los conocimientos obtenidos en programas educativos se representa a través de diplomas y certificados genéricos, que no profundizan detalles sobre cómo, cuándo y dónde se obtuvieron dichos conocimientos. Estos documentos usualmente indican datos básicos tales como el nombre del participante, el título del curso concluido, la fecha en la que se realizò y el nombre de la institución organizadora del programa. Debido a las obvias limitaciones físicas de estos documentos no es posible brindar más información sobre los contenidos del curso, la metodología empleada, los recursos de aprendizaje presentados y las habilidades que fueron evaluadas.

Un sistema que represente de manera específica el nivel de especialización y éxito obtenido por los trabajadores durante la culminación de programas formativos laborales, permitiría a los empleadores reconocer las habilidades y capacidades específicas de cada uno de ellos, facilitando así la toma de decisiones para una mejor selección de personal, promociones y ascensos. En un escenario ideal, este reconocimiento de habilidades podría replicarse en distintas empresas o corporaciones a nivel regional y nacional inclusive, si es que se brindan las condiciones logísticas y regulatorias necesarias, facilitando la movilidad laboral de los trabajadores.

En los últimos años hemos sido testigos de avances tecnológicos que han facilitado la consolidación de los medios de comunicación digitales como referentes para la transmisión de información, así como la mejora exponencial en la capaci- dad de almacenamiento de grandes cantidades de datos. También disponemos de interfaces de búsqueda que permiten la recuperación de información sistematizada que pueden ser consultadas desde múltiples dispositivos y puntos de acceso, y gracias a la masificación de redes sociales se han conformado grandes comunidades de pares en ámbitos sociales, laborales y académicos que facilitan la interacción e intercambio de ideas.

Según se indica en el documento publicado por la Fundación COTEC para la Innovación Tecnológica (2010), en el período comprendido entre 1997 y 2001 el desarrollo de las nuevas tecnologías de información y comunicación (TIC) obtuvo un impulso significativo debido a la popularización de internet. Al alcanzar progresivamente una masa crítica considerable de usuarios, las TIC han creado un nuevo entorno de aprendizaje no lineal que permite presentar la información de una manera diferente, conformando lo que se denomina una sociedad digital que justamente requiere de espacios educativos adaptados a estos cambios para poder satisfacer las nuevas demandas de información.

Las tecnologías de la información y la comunicación (TIC), por sus características y su rápida implantación a escala global, están provocando cambios significativos en muchos ámbitos y de manera especial en los institucionales. (...) La acumulación de información, la velocidad en su transmisión, la superación de las limitaciones o barreras espaciales, el empleo simultáneo de múltiples medios (imagen, sonido, texto, código) son, entre otros, los elementos que explican la enorme capacidad de cambio que aportan estas tecnologías (de Pablos, 2010, p.7).

De igual forma, esta nueva realidad tecnológica ha propiciado la evolución de los sistemas de evaluación y reconocimiento de logros no solamente en ámbitos comerciales y empresariales, sino también en actividades académicas, a través de la implementación de sistemas de insignias digitales.

Los sistemas de insignias digitales cuentan con la capacidad tecnológica y estructural para materializar los beneficios mencionados, gracias al correcto aprovechamiento de las características inherentes a las nuevas tecnologías: disponibilidad y acceso permanente a la información, transmisión y difusión global de contenidos, integración 
con recursos multimedia que facilitan el registro de actividades y extensa capacidad de almacenamiento de datos.

La utilización de símbolos físicos visuales, tales como medallas e insignias, ha sido una práctica común dentro de diversas instituciones y diversos ámbitos sociales (laborales, educativos, deportivos, etc.) para la identificación y reconocimiento de determinadas características asignadas o vinculadas a los individuos integrantes de una comunidad y/o sociedad. El uso de estos elementos también ha servido para comunicar pertenencia a una organización, establecer jerarquías, resaltar habilidades, así como, para visibilizar el reconocimiento de objetivos cumplidos ante un determinado grupo de pares (Halavais, 2012).

En su definición más genérica, las insignias digitales son una representación visual de un logro, competencia o habilidad, que tiene la particularidad de encontrarse disponible siempre en línea desde un sitio web y accesible en cualquier momento para su consulta o utilización. Las insignias digitales no solamente funcionan como credenciales, sino también permiten validar las habilidades enunciadas a través de los metadatos contenidos dentro de ellas. Estos metadatos ayudan a explicar el contexto, el proceso de evaluación y los resultados de una determinada actividad. Además, se encuentran vinculados a evidencia probatoria en forma de archivos de texto o material audiovisual, que servirá para validar las habilidades o logros que afirman haberse obtenido (Schwarz, 2016; Ostashewski y Reid, 2015).

Las insignias digitales se plantean como una nueva opción a los sistemas tradicionales de acreditación de logros educacionales basados en certificados y diplomas impresos, no solamente por las ventajas inherentes a los recursos electrónicos, sino también por su posible aplicación en áreas educativas no formales, en las cuales resulta necesario visibilizar competencias blandas o habilidades obtenidas por la experiencia social y/o laboral. Las insignias representan una alternativa real para incrementar la granularidad del proceso de evaluación; así como, ofrecer indicadores más precisos sobre el éxito académico, específicamente en la descripción de competencias (Ahn et al., 2014; Fanfarelli y McDaniel, 2015). Finalmente, un requisito importante de las insignias es la validez otorgada por instituciones de prestigio o expertos temáticos que acrediten la veracidad de la información proporcionada, además de la validez social, otorgada por la comunidad de usuarios que participan de los programas y admiten su valor como instrumento para el reconocimiento de logros (Hamari, 2015; Hurst, 2015).

En comparación con otros proyectos tecnológicos aplicados a temas educativos, aún no existen suficientes estudios sobre insignias digitales para determinar con exactitud su impacto definitivo en los procesos de enseñanza y aprendizaje. Las investigaciones que se han llegado a culminar evidencian el potencial de los sistemas de insignias para el incremento de la visibilidad y portabilidad de habilidades en distintos contextos, además de fomentar la motivación, la retención de conocimientos y el compromiso de los usuarios hacia las actividades desarrolladas. Se ha comprobado la utilidad de las insignias como estímulo para la participación de los estudiantes y también como elemento para fomentar un cambio de actitud positivo con respecto de los cursos dictados (Shields, 2017; Facey-Shaw et al., 2018). Las plataformas de insignias permiten establecer un nuevo punto de acceso para la comunicación directa entre los participantes del proyecto, lo cual resulta útil para conocer las necesidades específicas de los estudiantes para poder recibir una adecuada retroalimentación luego de la revisión de las evidencias de trabajo. La asesoría que puedan brindar los docentes responsables al estudiante, así como la facilidad de establecer un diálogo directo entre los actores del sistema, genera un ambiente propicio para el desarrollo de actividades prácticas relacionadas con las competencias blandas, cuya identificación y posterior evaluación no siempre encuentran un espacio adecuado (Hennah, 2017; Mah e Ifenthaler, 2018). Es necesario recalcar que uno de los factores de éxito de la aplicación de insignias digitales reside en un adecuado diseño de las insignias, de tal forma que los contenidos representados sean fácilmente identificables por todos los participantes del ecosistema y que además tengan correlación con las habilidades aprendidas. Las recompensas ofrecidas como consecuencia del uso de las insignias deben ser lo suficientemente atractivas para convencer a los estudiantes de la 
utilidad de estas. Los beneficios deben ser tangibles y representar verdaderamente una mejora para la carrera académica y/o profesional de los participantes, como por ejemplo la convalidación de cursos o la posibilidad de ascender laboralmente (Morris et al., 2019).

Dicho esto, resulta de gran interés conocer cómo las insignias digitales pueden servir de ayuda a los profesionales para la actualización y mejora de sus conocimientos laborales, especialmente en una época de alta especialización y competitividad. Las nuevas credenciales laborales elaboradas a partir de las insignias digitales permitirán mostrar con claridad las evidencias de que el trabajador tiene la capacidad de realizar las funciones asignadas, crear una identidad laboral digital que pueda ser compartida en plataformas de uso público o interno para consulta de sus pares y propiciar la formación de redes conformadas por individuos o entidades externas que establezcan nuevas políticas de evaluación (Borras-Gené, 2017).

Debido a las características descritas, la implementación de un sistema de insignias en un ambiente de trabajo facilitará la transición hacia una cultura de aprendizaje dentro de la organización. Aberdour (2016) señala incluso que las insignias pueden ser de gran utilidad en las siguientes tareas: reclutamiento de empleados, entrenamiento interno de la fuerza de trabajo, certificación de cumplimiento de estándares y obviamente en los programas de formación continua profesional.

Finalmente, Finkelstein, Knight y Manning (2013) mencionan algunos beneficios adicionales que pueden ofrecer las insignias para las empresas tales como el reconocimiento de la experiencia de vida de los trabajadores, es decir de aquellos logros adquiridos en ámbitos educativos no formales inclusive. Las insignias también facilitarían el proceso de reclutamiento interno y externo de trabajadores, puesto que los reclutadores tendrían acceso a una visión granular de las competencias y habilidades de los postulantes, pudiendo en todo caso encontrar al candidato más adecuado para el puesto requerido. Incluso es posible que las empresas inicien proyectos de learning analytics mediante los cuales puedan predecir qué empleados tienen mayores posibilidades de tener éxito en un determinado puesto basándose en el análisis de los datos disponibles, además de sugerir qué otras competencias podrían mejorar para cumplir con los requisitos del puesto.

El objetivo principal de la investigación es describir las tendencias de uso de los principios de diseño de insignias digitales en los programas de educación continua profesional. Para tal fin, se identificarán los tipos de uso de los principios de diseño de insignias digitales, referidos a reconocimiento, evaluación y motivación del aprendizaje durante el desarrollo de las actividades educativas descritas en los trabajos de investigación analizados.

\section{Método}

En el presente estudio se emplearon las técnicas propias de una revisión exploratoria scoping review, que es una forma de síntesis de conocimiento, que apunta a mapear la literatura existente dentro de un área temática en términos de volumen, naturaleza y características de investigación primaria, siempre buscando una respuesta a una pregunta de investigación genérica y amplia. El producto final de todo este proceso es una base de datos bibliográfica exhaustiva que ofrezca un perfil detallado de la literatura existente y que a su vez pueda servir como base para futuras revisiones mucho más complejas y rigurosas, como por ejemplo una revisión sistemática, o en su defecto, estos datos pueden ser utilizados también para determinar la viabilidad y pertinencia de una ampliación del estudio (Brien et al., 2010; Peterson et al., 2017; Pham et al., 2014; Munn et al., 2018).

Previamente se identificaron dos revisiones de literatura sobre insignias digitales ya publicadas, pero con un enfoque distinto al utilizado en el presente artículo. Con respecto al impacto de las insignias en relación con las actividades educativas orientadas al sector profesional, en la revisión realizada por Liyanagunawardena, Scalzavara y Williams (2018) se llegó a la conclusión de que la mayoría de los empleadores veían con interés el desarrollo de las insignias como una herramienta útil para conocer las habilidades de los potenciales trabajadores, facilitando la toma de decisiones. De igual manera, en la revisión publicada por 
Motheeram, Herselman y Botha (2019), se respalda la afirmación previa, puesto que para los empleadores las insignias representan un medio para simplificar la comunicación entre la academia, los futuros profesionales y el sector laboral, ya que permiten obtener información granular sobre los conocimientos y competencias disponibles en el mercado. Aunque ambas investigaciones decidieron emplear una cobertura general sobre el tema, el presente estudio es el primero en enfatizar las experiencias en programas de insignias digitales con características específicas tanto en audiencia, como en contenido curricular.

Las revisiones exploratorias ofrecen valor a los investigadores, los académicos de posgrado y los formuladores de políticas que desean establecer datos de referencia sobre la disponibilidad de investigación sobre un tema, o planificar futuras investigaciones y revisiones de otro tipo (Lockwood et al., 2019). También han resultado útiles cuando un tema es novedoso o no ha sido estudiado detenidamente, puesto que permite identificar de manera relativamente rápida los conceptos claves sobre el tema, así como cualquier vacío o temática pendiente de investigación, por tal motivo se adecúa perfectamente al tema de las insignias digitales.

Para el desarrollo de la revisión se utilizó la metodología de trabajo planteada por Arksey y O'Malley (2006), la cual consiste en seis etapas: planteamiento de la pregunta de investigación, identificación de los documentos, selección de los documentos, análisis de los datos recuperados, sumarización de resultados y consulta a los expertos, siendo esta última una etapa opcional.

Las revisiones exploratorias se caracterizan por responder a una pregunta de investigación gené- rica con base en la evaluación y análisis de la mayor cantidad de literatura publicada. La pregunta de investigación planteada es ¿cuáles son las tendencias sobre los principios de diseño para la aplicación de insignias digitales en los programas de educación continua profesional?

La finalidad de esta etapa del proceso era identificar investigaciones que brinden información detallada sobre el proceso de implementación de sistemas de insignias digitales en programas educativos de formación continua especializada dirigidas a profesionales pertenecientes a distintas carreras. Las investigaciones debían reportar detalles acerca del diseño curricular y temático de los cursos, las características técnicas de las plataformas de insignias utilizadas, el perfil profesional de la audiencia a la cual iba dirigida y en caso sea aplicable, mencionar los resultados y experiencias obtenidas luego de la aplicación del programa.

Se realizó una búsqueda de toda la bibliografía publicada en las bases de datos Scopus (entre los años 2012 y 2019) y Web of Science (entre los años 1985 y 2019), incluyendo artículos de revistas, capítulos de libros y ponencias en conferencias científicas. Se consideraron estas dos fuentes académicas debido a que las publicaciones registradas han sido sometidas a un proceso estricto de selección que asegura la calidad de los contenidos, así como la rigurosidad científica del proceso de investigación realizado.

En la primera etapa de selección de los documentos para analizar, se eliminaron las referencias bibliográficas que cumplían alguna de las siguientes condiciones: registros duplicados en ambas bases de datos, registros que hacen mención de una obra completa (libro o conferencia) y regis-

Tabla 1

Estructura de la Búsqueda Bibliográfica Realizada en las Fuentes Documentales

\begin{tabular}{|l|l|}
\hline \multicolumn{1}{|c|}{ Scopus } & \multicolumn{1}{|c|}{ Web of Science } \\
\hline \multicolumn{1}{|c|}{ Cadena de búsqueda: } & \multicolumn{1}{c|}{ Cadena de búsqueda: } \\
\hline $\begin{array}{l}\text { TITLE-ABS-KEY (“digital badges" OR “digital badging" OR } \\
\text { "microcredentials" OR “open badges") }\end{array}$ & $\begin{array}{l}\text { TEMA: (“digital badges" OR “digital badging” OR } \\
\text { "microcredentials" OR "open badges"). }\end{array}$ \\
\hline Período de tiempo: 2012-2019 & Período de tiempo: 1985-2019. \\
\hline $\begin{array}{l}\text { Colecciones revisadas: Scopus tiene una sola colección } \\
\text { general }\end{array}$ & $\begin{array}{l}\text { Colecciones revisadas: Science Citation Index Expanded } \\
\text { (SCIE), Social Sciences Citation Index (SSCI), Arts y } \\
\text { Humanities Citation Index (AHCI) }\end{array}$ \\
\hline
\end{tabular}


tros que no eran relevantes al estudio al no reportar experiencias sobre programas educativos dirigidos a la formación continua y desarrollo de profesionales. Adicionalmente, se revisaron los resúmenes de las investigaciones restantes para verificar que los contenidos estuvieran relacionados directamente con la temática propuesta para el estudio. Luego de aplicar los filtros mencionados, se obtuvo un total preliminar de cuarenta documentos.

Los documentos preseleccionados fueron categorizados de la siguiente manera:
Veinte investigaciones teóricas y/o empíricas que describen detalladamente las características técnicas y conceptuales de los proyectos de insignias digitales, así como los resultados de la aplicación de los mismos.

Quince documentos que no brindaban información sobre proyectos ya concluidos, sino más bien planteaban pautas y recomendaciones teóricas para la implementación y desarrollo de futuros proyectos de insignias digitales.

Tres artículos recopilatorios con breve infor-

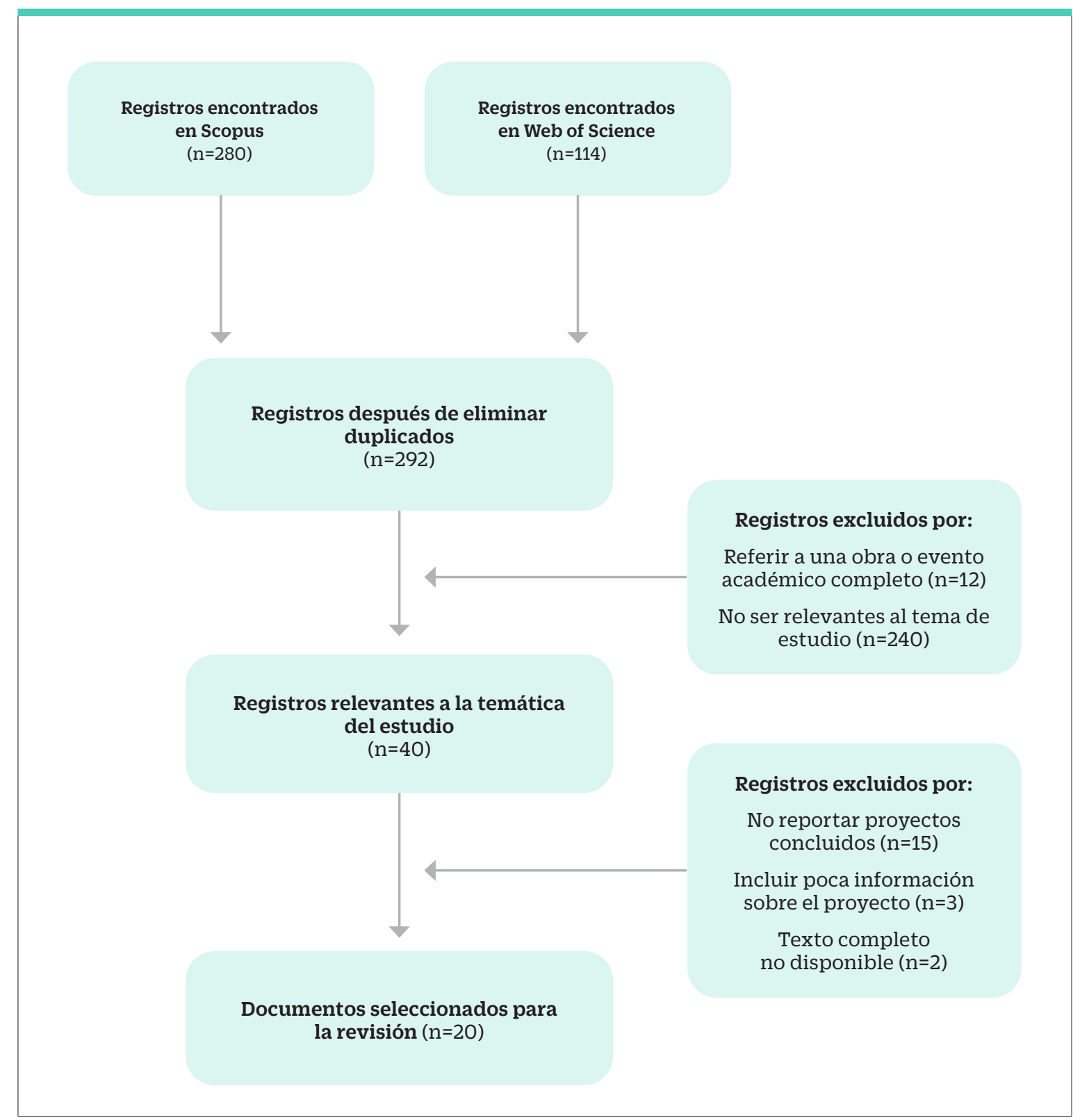

Figura 1. Diagrama de Flujo PRISMA Utilizado Para la Selección de Artículos 
mación general acerca de la existencia de proyectos ya implementados de insignias digitales. Básicamente se reportan los objetivos del proyecto, la temática de los contenidos y la audiencia a la que estaban dirigidos. No se incluyen detalles técnicos, ni resultados.

Dos artículos cuyo texto completo no fue posible conseguir por ningún medio (bases de datos académicas o redes internas de colaboración).

Finalmente se decidió analizar las experiencias descritas en los primeros veinte documentos, debido a que el detalle de la información proporcionada permitiría identificar una mayor cantidad de principios de diseño. Todo el proceso de selección fue sistematizado según los lineamientos PRISMA aplicables para trabajos de revisión de literatura (Tricco et al., 2018; Liberati et al., 2009).

\section{Resultados}

Los veinte documentos seleccionados reportaron un total de dieciséis proyectos de insignias digitales implementados en programas y cursos de formación continua profesional. Cabe resaltar que algunos de estos proyectos fueron mencionados más de una vez en distintas investigaciones.

La mayoría de los proyectos, once en total, fueron desarrollados por instituciones educativas y/o gubernamentales pertenecientes a Estados Unidos (68.75\%). Los cinco proyectos restantes se ejecutaron en Australia, Finlandia, Canadá, Italia y España respectivamente. No se encontraron experiencias registradas sobre insignias digitales en educación continua en países de América Latina,

Con respecto a las instituciones responsables cabe destacar que diez proyectos estuvieron a cargo en forma total de universidades o centros de educación superior (62.50\%), dos proyectos a cargo de entidades gubernamentales de alcance nacional, dos proyectos a cargo de organizaciones no gubernamentales y dos proyectos a cargo de consorcios o iniciativas mixtas conformadas por centros de investigación, universidades y otras entidades estatales.

Finalmente, once proyectos de insignias digitales (68.75\%) estuvieron orientados para capacitar docentes de cualquier nivel de enseñanza: educa- ción superior, educación primaria, instructores, etc. Seguidamente destacan tres proyectos dirigidos a profesionales e investigadores de distintas áreas de conocimiento: salud, negocios, tecnología, etc. Los dos proyectos restantes estuvieron enfocados a capacitación de personal administrativo en bibliotecas académicas.

Para el proceso de extracción de datos se tomaron como referencia las recomendaciones realizadas en el documento Design Principles Documentation Project (DPD), documento en el cual se describe un conjunto de buenas prácticas para el diseño general de sistemas de micro credenciales aplicadas en contextos educativos. La utilización de estos principios no es obligatoria, pero sí es altamente recomendable, puesto que proporcionan un punto de partida para la configuración de un ecosistema que permita aprovechar al máximo los beneficios de las insignias digitales en tanto se promueva un mayor compromiso de los participantes, que asegure la validez de los conocimientos representados y que también favorezca la difusión adecuada de toda la evidencia de aprendizaje obtenida.

Se enumeran en total veintinueve principios de diseño, organizados en tres categorías relacionadas directamente con las funcionalidades principales de las insignias digitales: reconocimiento, evaluación y motivación en el aprendizaje. La primera categoría está relacionada con facilitar el reconocimiento del aprendizaje mediante el incremento de la visibilidad de competencias y conocimientos, así como la validación de los mismos por parte de la comunidad educativa externa. La segunda categoría se enfoca en mejorar el proceso tradicional de evaluación y revisión de los productos de aprendizaje elaborados durante el desarrollo del programa de capacitación, gracias a la infraestructura tecnológica de las insignias digitales que permite la interacción de expertos, la difusión de los trabajos realizados y la integración con estándares y políticas. Finalmente, la tercera categoría se centra en utilizar insignias como elementos de motivación para el aprendizaje, es decir cómo estos nuevos elementos afectan la participación y colaboración entre los estudiantes para el cumplimiento de los objetivos educativos trazados (Hickey et al., 2014; Otto y Hickey, 2014).

Los principios de diseño se identificaron a tra- 
Tabla 2

Proyectos de Insignias Digitales Identificados

\begin{tabular}{|c|c|c|c|}
\hline Estudio & Proyecto & Institución responsable & País \\
\hline $\begin{array}{l}\text { Gamrat, Zimmerman, } \\
\text { Dudek y Peck (2014) } \\
\text { Gamrat y Zimmerman } \\
\text { (2015) } \\
\text { Gamrat y Zimmerman } \\
\text { (2016) }\end{array}$ & $\begin{array}{l}\text { Teacher Learning Journeys } \\
\text { (TLJ) }\end{array}$ & $\begin{array}{l}\text { Penn State University, NASA } \\
\text { y National Science Teachers } \\
\text { Association (NSTA) }\end{array}$ & Estados Unidos \\
\hline $\begin{array}{l}\text { Cucchiara, Giglio, Persico y } \\
\text { Raffaghelli (2014) }\end{array}$ & $\begin{array}{l}\text { Scientific Information for } \\
\text { Biomedical Research (SIBR) }\end{array}$ & Italian Digital Agenda & Italia \\
\hline $\begin{array}{l}\text { Epps, Kidds, Negro y Sayles } \\
\text { (2016) }\end{array}$ & $\begin{array}{l}\text { Customer Service Training } \\
\text { Curriculum Program }\end{array}$ & $\begin{array}{l}\text { University of Maryland } \\
\text { Libraries }\end{array}$ & Estados Unidos \\
\hline Kappes y Beltro (2015) & Open ACC Workshop & XSEDE & Estados Unidos \\
\hline Bondie (2015) & Project Reach Online & $\begin{array}{l}\text { US Department of } \\
\text { Education }\end{array}$ & Estados Unidos \\
\hline $\begin{array}{l}\text { Lius (2016) } \\
\text { Kullaslathi, Ruhalahti y } \\
\text { Brauer (2019) } \\
\text { Brauer, Korhonen y } \\
\text { Siklander (2019) }\end{array}$ & Learning Online PDP & $\begin{array}{l}\text { Omnia, The Joint Authority } \\
\text { of Education in Espoo, } \\
\text { Oulu University of Applied } \\
\text { Sciences, HAMK School } \\
\text { of Vocational Teacher } \\
\text { Education }\end{array}$ & Finlandia \\
\hline Diamond y Gonzalez (2016) & $\begin{array}{l}\text { Who Built America Project } \\
\text { (WBA) }\end{array}$ & $\begin{array}{l}\text { City University of New York } \\
\text { (CUNY) }\end{array}$ & Estados Unidos \\
\hline $\begin{array}{l}\text { Copenhaver y Pritchard } \\
\text { (2017) }\end{array}$ & $\begin{array}{l}\text { Eckerd College Library } \\
\text { Access Services Training } \\
\text { Program }\end{array}$ & Eckerd College & Estados Unidos \\
\hline Dyjur y Lindstrom (2017) & $\begin{array}{l}\text { Programa de capacitación } \\
\text { profesional no nombrado }\end{array}$ & $\begin{array}{l}\text { Un centro de educación } \\
\text { superior no nombrado }\end{array}$ & Canadá \\
\hline Jones, Hope y Adams (2018) & $\begin{array}{l}\text { Programa de capacitación } \\
\text { profesional no nombrado }\end{array}$ & $\begin{array}{l}\text { Una facultad clínica } \\
\text { universitaria }\end{array}$ & Estados Unidos \\
\hline Borras Gene (2018) & $\begin{array}{l}\text { Tele Education Office } \\
\text { (GATE) }\end{array}$ & $\begin{array}{l}\text { Universidad Politécnica de } \\
\text { Madrid }\end{array}$ & España \\
\hline Hunsaker y West (2019) & Tech with Kids & Brigham Young University & Estados Unidos \\
\hline Borup y Evmenova (2019) & Online Teaching Initiative & $\begin{array}{l}\text { Una universidad } \\
\text { estadounidense }\end{array}$ & Estados Unidos \\
\hline $\begin{array}{l}\text { Keane, Otter, Oxley y } \\
\text { Lipscomb (2016) }\end{array}$ & $\begin{array}{l}\text { VIF Professional } \\
\text { Development Program }\end{array}$ & $\begin{array}{l}\text { Visiting International } \\
\text { Faculty (VIF International } \\
\text { Education) }\end{array}$ & Estados Unidos \\
\hline $\begin{array}{l}\text { Dona, Gregory y Pechenkina } \\
\text { (2016) }\end{array}$ & Carpe Diem MOOC & $\begin{array}{l}\text { Swinburne University of } \\
\text { Technology }\end{array}$ & Australia \\
\hline $\begin{array}{l}\text { Goodrum, Abaci y Morrone } \\
\text { (2016) }\end{array}$ & Learning Technologies & Indiana University & EstadosUnidos \\
\hline
\end{tabular}


Tabla 3

Principios de Diseño con Mayor Frecuencia de Aparición

\begin{tabular}{|c|c|c|}
\hline Categoría & Principio & $\begin{array}{l}\text { Cantidad } \\
\text { de estudios }\end{array}$ \\
\hline Evaluación & Utilización de insignias jerarquizadas & 11 \\
\hline Reconocimiento & $\begin{array}{l}\text { Utilización de insignias como medio de comunicación de las habilidades } \\
\text { adquiridas }\end{array}$ & 10 \\
\hline Evaluación & Utilización de tareas basadas en rendimiento (performance assessment) & 9 \\
\hline Reconocimiento & $\begin{array}{l}\text { Elaboración de una ruta de aprendizaje que permita establecer objetivos y } \\
\text { visualizar avances }\end{array}$ & 7 \\
\hline Evaluación & $\begin{array}{l}\text { Retroalimentación constructiva de las actividades realizadas por parte de los } \\
\text { evaluadores }\end{array}$ & 5 \\
\hline Evaluación & Utilización de rúbricas & 5 \\
\hline Motivación & Utilizar distintos tipos de evaluaciones & 5 \\
\hline Reconocimiento & Utilización de estándares educativos para la elaboración de insignias y actividades & 3 \\
\hline Evaluación & Utilización de portafolios digitales & 3 \\
\hline Motivación & Utilización de insignias fácilmente identificables con roles o carreras & 3 \\
\hline Motivación & Promoción de la colaboración y el trabajo en equipo entre participantes & 3 \\
\hline Reconocimiento & Reconocimiento de distintos tipos de aprendizaje (competencias blandas y duras) & 2 \\
\hline Motivación & Interacción con miembros de la comunidad & 2 \\
\hline Reconocimiento & Respaldo de instituciones externas para otorgar validez a las insignias & 1 \\
\hline Reconocimiento & Participación de profesionales expertos para validar la asignación de insignias & 1 \\
\hline Reconocimiento & $\begin{array}{l}\text { Asignación de créditos académicos y otros privilegios como recompensa por la } \\
\text { obtención de insignias }\end{array}$ & 1 \\
\hline Motivación & Fomento del espíritu de competencia educativa & 1 \\
\hline
\end{tabular}

vés de la descripción del funcionamiento y aplicación de los sistemas brindada por los autores. Por ejemplo, en un proyecto se indicó que los productos académicos elaborados por los participantes fueron revisados y corregidos por expertos profesionales, además de los docentes encargados del curso. Si la decisión de los expertos era favorable, se asumía el curso como aprobado y se procedía con la asignación de la insignia. Además, los organizadores del proyecto habían establecido previamente convenios con otras instituciones para que revisen el perfil digital de los participantes en donde se mostraban las insignias para que sean tomados en cuenta para futuros concursos de selección de personal. De acuerdo con la información brindada, se asumió que en el proyecto se utilizaron los siguientes principios: "Respaldo de instituciones externas para otorgar validez a las insignias" y "Participación de profesionales expertos para validar la asignación de insignias".

El principio más utilizado fue el diseño de insignias agrupadas en categorías jerárquicas, de tal forma que, para obtener las insignias de mayor valor académico, resulta necesario cumplir una serie de requisitos previos establecidos por los diseñadores del sistema. Las insignias ubicadas en las categorías inferiores no requieren de mucha exigencia para su obtención, usualmente se asignan al demostrar la asistencia del participante en algún seminario en línea o sesión informativa, o con la redacción de un breve informe. Conforme quiera obtener las insignias de categorías más avanzadas, la dificultad para la obtención aumenta, en algunos casos se solicita elaborar propues- 
tas para la aplicación práctica de los temas aprendidos. En los proyectos revisados se establecieron hasta tres categorías para agrupar las insignias, las cuales fueron denominadas de manera distinta de acuerdo con cada experiencia, pero la idea general en todos los casos era representar tres niveles fijos de dificultad de obtención; básico, medio y avanzado. En ocasiones se otorgaba una insignia adicional por superar una cantidad mínima de cursos disponibles en algunos de los tres niveles, la cual representaba haber obtenido dominio sobre determinado tema (Cucchiara et al,. 2014; Gamrat et al., 2014; Gamrat y Zimmerman, 2015; Gamrat et al., 2016; Kappes y Betro, 2015; Diamond y Gonzalez, 2016; Goodrum et al., 2016; Keane et al., 2016; Dona et al., 2016; Jones et al., 2018; Borup y Evmenova, 2019; Lius, 2016; Brauer et al., 2019; Kullaslahti et al., 2019; Hunsaker y West, 2020).

El segundo principio con mayor frecuencia fue la utilización de insignias digitales como medio de comunicación, a través del cual los participantes tienen la oportunidad de mostrar a los miembros de su comunidad las habilidades y conocimientos aprendidos. Ellos tienen la potestad de decidir qué insignias mostrar, por cuánto tiempo y quiénes tienen autorización para visualizarlas. Los participantes tuvieron la oportunidad de difundir y compartir sus logros desde un espacio virtual dentro del perfil de usuario proporcionado por la plataforma, o también a través de redes sociales. En algunos casos podían integrar las insignias dentro de su currículum en formato electrónico. Cabe mencionar que esta funcionalidad debe ser explicada debidamente para promover su aprovechamiento. En algunos proyectos el porcentaje de usuarios que no conocían o no estaban interesados en visibilizar sus logros resultó sorprendentemente alto (Cucchiara et al., 2014; Gamrat et al., 2014; Gamrat and Zimmerman, 2015; Gamrat et al., 2016; Bondie, 2015; Goodrum et al., 2016; Keane et al., 2016; Borrás-Gené, 2018; Dyjur y Lindstrom, 2017; Jones et al., 2018).

El sistema de insignias digitales debe permitir al estudiante la posibilidad de elaborar su propia ruta de aprendizaje, entendida como una secuencia ordenada y sistematizada de cursos o actividades educativas que el alumno debe completar con la finalidad de obtener determinados conocimientos. Se analizaron siete estudios que implementa- ron una serie de funcionalidades orientadas a facilitar esta tarea: poner a disposición una amplia cantidad de actividades para no limitar la oferta de selección (Lius, 2016, Brauer, 2019, Kappes y Betro, 2015), permitir a los participantes establecer sus propios objetivos y metas de acuerdo con sus intereses profesionales (Gamrat, 2014, Bondie, 2015) así como brindar información completa y detallada de los contenidos del curso para propiciar una selección rigurosa. Asimismo, los participantes debían tener no solamente la posibilidad de monitorear los progresos de su aprendizaje, sino agregar o retirar cursos de acuerdo con sus intereses y plazos y tiempos para concluirlos (Cucchiara, 2014).

Otro principio identificado fue la utilización de distintos tipos de tareas basadas en el desempeño del estudiante para definir la obtención de las insignias. Los participantes debieron resolver satisfactoriamente distintos tipos de pruebas, no solamente exámenes o tests en línea, sino también redactar asignaciones, elaborar materiales para el dictado de cursos, diseñar objetos multimedia, elaborar proyectos aplicativos, etc. Incluso se consideraba en ocasiones la asignación de insignias por participación, que toman en cuenta la asistencia participativa en actividades previamente programadas (discusiones asíncronas en foros, charlas en línea, etc.) como parte de la evaluación. Una amplia oferta de productos académicos permite un proceso de evaluación granular y con mayor precisión para determinar el nivel de éxito del estudiante (Cucchiara et al., 2014; Gamrat et al., 2014; Gamrat and Zimmerman, 2015; Gamrat et al., 2016; Bondie, 2015; Goodrum et al., 2016; Keane et al., 2016; Kappes and Betro, 2015; Diamond y Gonzalez, 2016; Dona et al., 2016; Dyjur y Lindstrom, 2017).

Proporcionar a los participantes retroalimentación y comentarios formativos acerca de su desempeño en los cursos representa un aporte significativo para la culminación exitosa de los cursos. Usualmente los docentes especialistas trabajaban con un tipo de tutoría, asesorando a los participantes y revisando los productos de enseñanza, indicando sugerencias para mejorar los resultados. Los participantes tenían la posibilidad de responder a estos comentarios propiciando de esta manera discusiones que permitían la construcción de conocimientos que apuntaban a obtener 
la insignia con el nivel máximo posible de excelencia (Gamrat et al., 2014; Gamrat y Zimmerman, 2015; Gamrat et al., 2016; Bondie, 2015; Diamond y Gonzalez, 2016; Borup y Evmenova, 2019; Lius, 2016; Brauer et al., 2019; Kullaslahti et al., 2019).

En cinco estudios se emplearon distintas formas para evaluar los resultados de aprendizaje tales como la corrección automatizada de exámenes por computadora o la revisión de trabajos por parte de otros estudiantes del curso. También se consideró la evaluación de los trabajos por parte de un docente experto que hacía las veces de tutor de los estudiantes a lo largo del curso. Cabe mencionar que en ningún estudio se estableció algún tipo de autoevaluación o autocrítica (Cucchiara et al., 2014; Gamrat et al., 2014; Gamrat y Zimmerman, 2015; Gamrat et al., 2016; Diamond y Gonzalez, 2016; Goodrum et al., 2016; Borrás-Gené, 2018).

Tres estudios utilizaron estándares de competencias y habilidades validados previamente dentro del ámbito académico y/o educativo. De esta forma, el diseño de los sistemas de insignias obtiene un sustento profesional reconocido por la comunidad, además que los contenidos representados por las insignias pueden resultar mucho más familiares a los participantes. Los proyectos estudiados utilizaron diversos tipos de estándares educativos: gubernamentales de alcance nacional (Brauer, 2019), internacionales (Borup y Evmenova, 2019). Incluso en algunos casos se tuvo la necesidad de elaborar lineamientos propios, tomando como punto de partida hasta cinco estándares distintos (Bondie, 2015).

El uso de portafolios digitales como herramienta complementaria se reportó en tres casos. Los portafolios digitales (e-portfolios) son plataformas en línea donde los participantes pueden almacenar los materiales, documentos y evidencias elaborados por ellos durante el desarrollo del curso, de tal forma que puedan demostrar ante la comunidad todos los logros obtenidos. Estos portafolios usualmente son de acceso público, aunque los participantes pueden decidir el nivel de acceso a los contenidos (Cucchiara et al., 2014; Gamrat et al., 2014; Gamrat y Zimmerman, 2015; Gamrat et al., 2016; Keane et al., 2016).

Se recomienda que las insignias tengan una denominación familiar, cuyo nombre sea lo suficientemente descriptivo para que los participantes puedan asociar fácilmente a un rol, puesto o cargo de su interés. Es importante que estos nombres tengan algún tipo de correlación con títulos reales y que además brinden algún tipo de información sobre las habilidades que conllevan dicho puesto, como por ejemplo Master Builder, Journeyman Builder, Apprentice Builder, TechGuru, Common Core Writing Specialist, History Geek. Insignias de otros proyectos aludían no solamente al puesto, sino también al nivel de dominio alcanzado como Specialist Expert o Social Media Novice (Cucchiara et al., 2014; Diamond y Gonzalez, 2016; Lius, 2016; Brauer et al., 2019; Kullaslahti et al., 2019).

Se otorgan insignias por actividades que priorizan la obtención de méritos grupales o que promueven el trabajo en equipo. Esta situación ocurre cuando todas las actividades del programa se enfocan en promover el trabajo colaborativo a través de seminarios o discusiones en línea. El objetivo era facilitar a los participantes a que propongan sus conocimientos mediante el contacto con sus pares para conseguir una experiencia de aprendizaje compartida. Las insignias se convertían en un medio para motivar a los participantes en establecer una conexión sólida con sus compañeros facilitando espacios para la comunicación, colaboración y cooperación. Otros proyectos conformaban grupos de trabajo de cuatro o cinco personas para que interactuaran regularmente en foros de discusión y pudieran compartir sus expectativas e ideas con respecto al curso, así como otras actividades colaborativas, tales como la revisión de trabajos presentados o conversaciones para liberar tensiones, con la finalidad de conformar una comunidad de aprendizaje confiable (Gamrat et al., 2014; Gamrat y Zimmerman, 2015; Gamrat et al., 2016; Diamond y Gonzalez, 2016; Borup y Evmenova, 2019).

Se debe permitir la creación de insignias que reconozcan también habilidades blandas tales como liderazgo, empatía, comunicación interpersonal, capacidad para trabajo en equipo, etc. Estas competencias por lo general no son tomadas en cuenta por los sistemas de evaluación tradicionales, motivo por el cual el uso de las insignias digitales representa una oportunidad valiosa para otorgarles visibilidad (Cucchiara, 2014, Bondie, 2015).

Se otorgan insignias si durante el proceso de aprendizaje los participantes interactúan con co- 
legas o miembros de la comunidad, tal como en el proyecto Who Built America, en el cual se otorgan "Community Badges" cuando se ha contribuido con la comunidad profesional en general, a través del intercambio de materiales de enseñanza, ofreciendo retroalimentaciones o comentando en los foros (Diamond y Gonzalez, 2016; Lius, 2016; Brauer et al., 2019; Kullaslahti et al., 2019).

La participación de profesionales expertos durante el proceso de asignación de insignias es un valor añadido necesario para reforzar la validez de las habilidades y conocimientos aprendidos. El proyecto Teacher's Learning Journey, dirigido a capacitar docentes especialistas en las áreas de ciencias naturales, contó con el apoyo de trabajadores profesionales de la NASA quienes se encargaban de asesorar y aconsejar a los participantes durante el desarrollo de cursos. Los cursos trataban temas sobre ingeniería, medio ambiente y astronomía; la culminación exitosa de los mismos era evaluada por el experto quien finalmente decidía si era pertinente la asignación de la insignia (Gamrat et al., 2014; Gamrat y Zimmerman, 2015; Gamrat et al., 2016).

Al igual que la participación de expertos, la obtención del reconocimiento de entidades externas al proyecto incrementa las posibilidades de aprovechamiento de las insignias digitales. Para tal fin, la institución responsable del proyecto debe realizar las coordinaciones y convenios necesarios con los empleadores interesados para que inicien el reconocimiento formal de las insignias obtenidas por los participantes. Solamente el proyecto VIF Professional Development Program consiguió obtener el apoyo de los administradores educativos estatales, quienes usualmente consultan la base de datos de participantes para monitorear el desempeño obtenido por los docentes y eventualmente tomarlos en cuenta para alguna oportunidad laboral. Además, este proyecto consiguió que las escuelas pertenecientes al distrito acordaran reconocer un total de diez horas de desarrollo profesional por cada curso completado exitosamente, de tal forma que las insignias tuvieran alguna equivalencia en el creditaje formal de los docentes (Keane, 2016).

Finalmente, para fomentar el espíritu de competencia entre los participantes, se diseñaron sistemas de calificaciones y rankings abiertos al pú- blico para estimular la obtención de insignias. Por ejemplo, en el proyecto Learning Online, conforme se realizaban progresos en el curso, se publicaba una lista de todas las insignias obtenidas por los participantes, como una manera de fomentar la competición entre los grupos de trabajo (Lius, 2016; Brauer et al., 2019; Kullaslahti et al., 2019).

\section{Discusión}

En primer lugar, luego de revisar los documentos seleccionados, se observa claramente que la mayoría de las experiencias reportadas se llevaron a cabo en Estados Unidos y en varios países de Europa. Es necesario mencionar que, en el caso de América Latina, la Pontificia Universidad Javeriana (Colombia) y el Tecnológico de Monterrey (México) ya han comenzado a ofrecer insignias digitales como certificaciones de aprendizaje en varios de sus programas de educación continua profesional. Como era de esperar, las insignias cumplen con incluir los metadatos básicos: nombre del curso, nombre del estudiante, fecha de obtención, descripción del curso y habilidades aprendidas. Las insignias se pueden distribuir en distintos medios, incluyendo correo electrónico y pueden compartirse en redes sociales como Facebook, Twitter o LinkedIn. También se brinda la oportunidad de publicarlas en sitios web, blogs e incluso generar un código QR para un acceso mucho más rápido. (Posgrados y Educación Continua, 2017; Educación Continua y Consultorías Javeriana, 2019). No se han encontrado publicaciones o estudios adicionales sobre los resultados de esta implementación que permitan evaluar el impacto en los planes educativos de ambas instituciones o la recepción por parte de los usuarios, solamente se cuenta con la información proporcionada por los canales de comunicación institucionales de ambas universidades.

Aparte de la distribución geográfica de los proyectos estudiados, es necesario mencionar que la gran mayoría de ellos tuvieron como público objetivo a docentes de educación básica y superior, investigadores en ciencias y personal administrativo de universidades. No se encontraron experiencias concretas dirigidas para profesionales 
de otras áreas. Esta situación ya ha sido advertida por Roy y Clark (2019), que indican una alta concentración de proyectos de insignias digitales en contextos mayormente académicos en CTM (ciencia, tecnología y medicina), ciencias sociales y humanidades. Existe entonces un mercado potencial aún no explorado en otras áreas profesionales en donde se podría aplicar y dar a conocer la utilidad de las insignias y microcredenciales.

Con respecto a la respuesta de los empleadores hacia la efectividad de estos programas, se puede hablar de una recepción mayormente positiva, situación que coincide con los hallazgos efectuados por Raish y Rimland (2016) cuyo estudio determinó que la gran mayoría de empleadores estaba de acuerdo en utilizar insignias digitales para la identificación de habilidades, siempre y cuando se cuente con información detallada y precisa acerca del funcionamiento del sistema. Además, resulta necesario incrementar la cantidad de instituciones y empresas que participen en programas de insignias, de tal manera que exista una masa crítica de participantes que intercambien información y experiencias con respecto a esta nueva tecnología.

Por ende, para poder diseñar un programa eficiente de incentivos laborales, tales como ascensos o bonificaciones salariales a sus trabajadores, es necesario que los empleadores puedan conocer al detalle las habilidades y conocimientos que los trabajadores hayan podido adquirir dentro de espacios de educación formal o desde la experiencia obtenida durante el desarrollo de las tareas asignadas. La posibilidad de incrementar la visibilidad de estas competencias a través de la publicación de las insignias digitales en redes sociales o plataformas de trabajo interno es tal vez la característica más atractiva de todo el sistema, por ello no resulta extraño que sea el principio con mayor frecuencia de implementación en todas las experiencias. Aunque los participantes tienen la opción de hacer públicos sus logros dentro de toda la comunidad académica global, el principal objetivo de esta tarea de difusión será siempre los empleadores o encargados de recursos humanos de las empresas, quienes son los que toman las decisiones al respecto de promociones o movilidad laboral.

La utilización de insignias jerarquizadas permite una mejor identificación y estructuración de las habilidades aprendidas, pues al establecer distintos niveles de insignias es posible determinar con exactitud el nivel de aprendizaje que han obtenido los participantes. Además, en el caso que el sistema permita cierta flexibilidad al momento de escoger los temas en los que se desea obtener especialización, los participantes podrán adecuar el desarrollo del curso a sus expectativas laborales y objetivos trazados. Esta característica también fue una de las más utilizadas en las experiencias revisadas y mantiene una relación estrecha con el aspecto de la visibilidad mencionado anteriormente: es posible incrementar la granularidad para la identificación de habilidades requeridas por los empleadores, facilitando la creación de un perfil profesional que encaje con los requerimientos laborales, incluyendo aquellas habilidades que tradicionalmente son difíciles de evaluar.

La validación de los contenidos aprendidos se otorga a través de tres instancias: evaluación de resultados de aprendizaje por parte de expertos profesionales, respaldo institucional de las organizaciones involucradas (educativas o corporativas), y finalmente la utilización de estándares educativos y/o rúbricas. Para que las insignias sean tomadas en cuenta como reemplazo adecuado de las certificaciones tradicionales, resulta necesario construir una red de confianza académica que facilite la aceptación del valor verdadero de los contenidos que son representados por las insignias. Además, queda implícita la participación de equipos multidisciplinarios conformados por profesionales con experiencia en diversas áreas para construir un sistema óptimo a todos los niveles: diseño, usabilidad, contenidos y respaldo técnico. Es necesario fomentar la colaboración de ingenieros, educadores, bibliotecarios, diseñadores gráficos y especialistas temáticos de acuerdo con los contenidos de los cursos que se deseen impartir, con la finalidad de presentar una oferta educativa atractiva tanto para los trabajadores como para las instituciones interesadas.

La implementación de una ruta de aprendizaje que ayude a monitorear los avances del participante y el cumplimiento de objetivos trazados fue una característica utilizada en pocos estudios. De igual forma, el reconocimiento de habilidades blandas durante los cursos dictados no fue una característica prioritaria durante el desarrollo de las actividades. Esta situación resulta poco usual, 
pues justamente ambas son funcionalidades exclusivas de los sistemas de insignias digitales y en teoría deberían aportar de manera significativa al proceso de enseñanza y producción de conocimientos. Una posible razón de este bajo nivel de incidencia puede ser la carencia de información sobre el funcionamiento de los sistemas de insignias. Al ser una nueva tecnología, es necesario brindar a los usuarios la mayor cantidad de guías, tutoriales y servicios de ayuda en línea que les permita aprender a utilizar y explotar al máximo los beneficios que pueden obtener de las insignias digitales. En caso de no contar con un servicio de tutoría informativa, muchas funcionalidades pueden ser no comprendidas por los estudiantes y por ende no aprovechar al máximo las características que ofrece la plataforma.

Los principios de diseño más utilizados fueron aquellos enfocados al reconocimiento y visibilidad de competencias, situación fácilmente explicada por el interés de los participantes en dar a conocer las habilidades laborales disponibles, a manera de un currículum en línea, verificado por instituciones confiables y en continua actualización. En cuanto a los principios centrados en el proceso de motivación para conseguir los objetivos planteados durante el aprendizaje, es posible que adquieran mayor relevancia cuando existe mayor oferta de recompensas tangibles relacionadas directamente con la realidad laboral dentro de la organización.

Hickey y Chartrand (2020) afirman que los programas de insignias digitales obtienen mayores probabilidades de éxito cuando se cumplen las siguientes condiciones: mantener expectativas reales sobre el alcance de la evaluación de las competencias de los estudiantes que permitan una administración eficiente de los resultados académicos; fomentar el aprendizaje y reconocimiento en una dimensión social adecuada que favorezca el diálogo e intercambio de ideas entre los participantes; concretar la asignación de insignias una vez que se haya verificado la culminación correcta de cursos, talleres y proyectos a través de la presentación de objetos de aprendizaje tangibles y no premiar únicamente la asistencia o participación. La aplicación de las tendencias mencionadas en el presente estudio favorece la implementación de estos escenarios y resultan un punto de partida básico para cualquier proyecto de reconocimiento y uso de microcredenciales, en especial cuando se trata de una tecnología relativamente nueva para el contexto regional.

En el presente estudio se realizó la revisión y análisis de los parámetros en el diseño de sistemas de insignias digitales aplicados en programas de formación continua profesional. Las fuentes revisadas fueron los resultados de investigaciones sobre programas dirigidos hacia la actualización y mejora de carreras dictadas a nivel técnico - profesional. Las investigaciones fueron publicadas en revistas indizadas en las bases de datos Web of Science y Scopus hasta el tercer trimestre del año 2019. Tal y como se mencionó anteriormente, existe un evidente sesgo geográfico al no contar con datos sobre experiencias en países latinoamericanos.

A mediano plazo se espera disponer de una mayor cantidad de investigaciones empíricas sobre insignias digitales, que permitan realizar nuevas revisiones sistemáticas y metaanálisis con la finalidad de evaluar y validar estadísticamente los hallazgos y evidencias producidos por dichos estudios.

\section{Recomendaciónes}

Toda implementación tecnológica requiere no solamente de conseguir los recursos económicos requeridos, tanto para la adquisición de hardware adecuado como para el diseño o compra del software adecuado. Es necesario tomar en cuenta si la comunidad de usuarios que será beneficiada con esta implementación se encuentra en capacidad de aprovechar los recursos informáticos a los cuales tendrán acceso. Con mayor razón si dicha implementación es relativamente nueva, se incrementa el riesgo que no se aproveche al máximo su potencial.

Las insignias digitales proporcionan a los responsables de la gestión de recursos humanos, una herramienta adicional para facilitar la toma de decisiones a nivel de recursos humanos. En cuanto a los trabajadores, tienen ahora la posibilidad de construir un portafolio digital que puede servir como vitrina de exposición durante la búsqueda 
de mejoras laborales de cualquier índole. Con la finalidad de garantizar condiciones adecuadas para un correcto desarrollo de un proyecto tecnológico novedoso y disruptivo, se plantea tomar en cuenta las siguientes observaciones:

Convencer a las autoridades y/o directivos de la importancia de invertir en programas de formación profesional continua como una herramienta para incrementar el bienestar del trabajador y por ende favorecer el clima organizacional de manera positiva. Es importante que el trabajador observe que su empresa se preocupa por su formación y desarrollo profesional, con programas y planes adecuados a sus objetivos. Las insignias digitales tienen la capacidad de identificar gradualmente las competencias que el trabajador requiere reforzar, así como aquellas que pueden servir para labores similares.

El diseño de los programas de insignias digitales requiere de la participación de un equipo multidisciplinario integrado por educadores, programadores, diseñadores, bibliotecarios, administradores en recursos humanos, técnicos y especialistas temáticos. Cada uno de estos profesionales desde su carrera pueden aportar para la construcción de una interfaz amigable, con contenidos revisados e información instructiva adecuada, alineados a favorecer no solamente a los trabajadores sino también a los fines de la empresa.

Es necesario estructurar los cursos en función de las competencias y habilidades identificadas por las empresas como relevantes para cumplir con los objetivos trazados por cada área. Se requiere establecer un canal de comunicación fluido entre las instancias responsables.

Es necesario mencionar que el éxito de estos programas depende también de un programa de inducción al uso de la plataforma debidamente detallado y explicado. Las insignias digitales son una nueva tecnología y es probable que la mayoría de los participantes desconozca su utilidad real. Los programas estudiados han incluido documentación, manuales de ayuda y asesoramiento en línea que permitió a los participantes aprender a utilizar la plataforma y conocer los beneficios. Caso contrario, es probable que las herramientas no sean utilizadas de manera adecuada y se genere confusión entre los participantes del curso.

La coyuntura actual de confinamiento y aisla- miento social establecida por razones sanitarias en la gran mayoría de países ha ocasionado el cierre temporal de espacios físicos y la cancelación de eventos que requieran contacto físico. Mientras no sea posible determinar con certeza cuando se llevará a cabo el retorno a la "normalidad", actividades como el teletrabajo y la educación a distancia se han tenido que implementar a gran escala con distintos niveles de eficacia. Como consecuencia de ello, las empresas se han visto en la necesidad de priorizar una serie de competencias en sus trabajadores que se requieren para afrontar este nuevo escenario a mediano y largo plazo: creatividad, innovación, pensamiento crítico, inteligencia emocional liderazgo y competencias digitales. Las empresas deben entonces diseñar planes de capacitación cuyo objetivo sea afianzar estas competencias blandas, e incluso se sugiere gestionar presupuestos destinados al aprendizaje, como prioridad en el plan estratégico. Mientras más rápido se establezcan procedimientos para integrar y desarrollar habilidades cognitivas, digitales, sociales y emocionales en la fuerza de trabajo, las empresas obtendrán una respuesta mucho más rápida ante cualquier innovación o rediseño de procesos laborales, enmarcados en un ámbito totalmente digital. (Agrawal, et al., 2020; Marr, 2020).

El escenario es propicio para relanzar los programas de formación continua como un elemento esencial y vital para las organizaciones. Como ya se ha demostrado las insignias digitales tienen la capacidad de identificar, promover y visibilizar estas competencias blandas, no tradicionales en los esquemas de evaluación actuales, y que actualmente resultarían de vital importancia para adaptarse a la nueva realidad digital.

\section{Referencias}

Aberdour, M. (2016). Transforming workplace learning culture with digital badges. En Foundation of Digital Badges and Micro-Credentials: Demonstrating and Recognizing Knowledge and Competencies (pp. 203219). https://doi.org/10.1007/978-3-319-15425-1_11

Agrawal, S., De Smet, A., Lacroix, S., y Reich, A. (2020, julio 
5). To emerge stronger from the COVID-19 crisis, companies should start reskilling their workforces now. McKinsey \& Company. https://www.mckinsey. com/business-functions/organization/ourinsights/to-emerge-stronger-from-the-covid-19crisis-companies-should-start-reskilling-theirworkforces-now\#

Ahn, J., Pellicone, A., y Butler, B. S. (2014). Open badges for education: what are the implications at the intersection of open systems and badging? Research in Learning Technology, 22(1), 23563. https://doi.org/10.3402/rlt.v22.23563

Arksey, H., y O'Malley, L. (2005). Scoping studies: towards a methodological framework. International Journal of Social Research Methodology, 8(1), 19-32. https:// doi.org/10.1080/1364557032000119616

Bondie, R. (2015). A digital teaching platform to further and assess use of evidence-based practices. Rural Special Education Quarterly, 34(1), 23-29. https:// doi.org/10.1177/875687051503400106

Borrás-Gené, O. (2017). Insignias digitales como acreditación de competencias en la Universidad. Universidad Politécnica de Madrid.

Borrás-Gené, O. (2018). Use of digital badges for training in digital skills within higher education. 2018 International Symposium on Computers in Education (SIIE), 1-7. https://doi.org/10.1109/ SIIE.2018.8586734

Borup, J., y Evmenova, A. S. (2019). The Effectiveness of Professional Development in Overcoming Obstacles to Effective Online Instruction in a College of Education. Online Learning, 23(2), 1-20. https://doi.org/10.24059/olj.v23i2.1468

Brauer, S., Korhonen, A.-M., y Siklander, P. (2019). Online scaffolding in digital open badge-driven learning. Educational Research, 61(1), 53-69. https://doi.org/ 10.1080/00131881.2018.1562953

Brien, S. E., Lorenzetti, D. L., Lewis, S., Kennedy, J., y Ghali, W. A. (2010). Overview of a formal scoping review on health system report cards. Implementation Science: IS, 5, 2. https://doi.org/10.1186/1748-5908-5-2

Copenhaver, K., y Pritchard, L. (2017). Digital badges for staff training: Motivate employees to learn with micro-credentials. Journal of Electronic Resources Librarianship, 29(4), 245-254. https://doi. org/10.1080/1941126X.2017.1378543

Cucchiara, S., Giglio, A., Persico, D., y Raffaghelli, J. E. (2014). Supporting self-regulated learning through digital badges: A case study. En Lecture Notes in
Computer Science (including subseries Lecture Notes in Artificial Intelligence and Lecture Notes in Bioinformatics) (Vol. 8699, pp. 133-144). https://doi. org/10.1007/978-3-319-13296-9_15

Curran, V., Gustafson, D. L., Simmons, K., Lannon, H., Wang, C., Garmsiri, M., Fleet, L., y Wetsch, L. (2019). Adult learners' perceptions of self-directed learning and digital technology usage in continuing professional education: An update for the digital age. Journal of Adult and Continuing Education, 25(1), 74-93. https://doi.org/10.1177/1477971419827318

de Pablos, J. (2010). Universidad y sociedad del conocimiento. Las competencias informacionales y digitales. Revista de Universidad y Sociedad del Conocimiento (RUSC), 7(2). https://doi.org/10.7238/ rusc.v7i2.977

Diamond, J., y Gonzalez, P. C. (2016). Digital badges for professional development: Teachers' perceptions of the value of a new credentialing currency. En Foundation of Digital Badges and MicroCredentials: Demonstrating and Recognizing Knowledge and Competencies (pp. 391-409). https://doi.org/10.1007/978-3-319-15425-1_21

Dona, K. L., Gregory, J., \& Pechenkina, E. (2016). Digital Badges as a Motivator in MOOCS: The Carpe Diem MOOC Experience. En L. Y. Muilenburg \& Z. L. Berge (Eds.), Digital Badges in Education: Trends, Issues and Cases (pp. 238-248). Routledge.

Dyjur, P., y Lindstrom, G. (2017). Perceptions and Uses of Digital Badges for Professional Learning Development in Higher Education. TechTrends, 61(4), 386-392. https://doi.org/10.1007/s11528-0170168-2

Educación Continua y Consultorías Javeriana. (2019, junio 7). Insignias Digitales Pontificia Universidad Javeriana. https://www.youtube.com/ watch?v=LFgNyv8tamo

Epps, S. K., Kidd, J., Negro, T., y Sayles, S. L. (2016). Rethinking customer service training: A curricular solution to a familiar problem. Journal of Access Services, 13(3), 199-209. https://doi.org/10.1080/153 67967.2016.1206476

Facey-Shaw, L., Specht, M., van Rosmalen, P., Börner, D., y Bartley-Bryan, J. (2018). Educational Functions and Design of Badge Systems: A Conceptual Literature Review. IEEE Transactions on Learning Technologies, 11(4), 536-544. https://doi. org/10.1109/TLT.2017.2773508

Fanfarelli, J. R., y McDaniel, R. (2015). Individual 
Differences in Digital Badging: Do Learner Characteristics Matter? Journal of Educational Technology Systems, 43(4), 403-428. https://doi. org/10.1177/0047239515588165

Finkelstein, J., Knight, E., y Manning, S. (2013). The Potential and Value of Using Digital Badges for Adult Learners. American Institutes for Research (AIR).

Fundación COTEC para la Innovación Tecnológica (Ed.). (2014). Educación digital y cultura de la innovación.

Gamrat, C., y Zimmerman, H. T. (2015). An online badging system supporting educators' STEM learning. En D. Hickey, J. Jovanovic, S. Lonn, y J. E. Willis III (Eds.), Proceedings of the Open Badges in Education (OBIE 2015) Workshop (Vol. 1358).

Gamrat, C., y Zimmerman, H. T. (2016). Teacher learning journeys: A design case study of a learner-centered stem digital badging system. En Digital Badges in Education: Trends, Issues, and Cases (pp. 215-225). Routledge, New York.

Gamrat, C., Zimmerman, H. T., Dudek, J., y Peck, K. (2014). Personalized workplace learning: An exploratory study on digital badging within a teacher professional development program. En British Journal of Educational Technology. https://doi. org/10.1111/bjet.12200

Gil López, A. J., y Gallego Gil, D. J. (2016). La realización de formación continua desde la perspectiva de la organización de aprendizaje. Educar, 52(1), 107-126. https://doi.org/10.5565/rev/educar.701

Goodrum, D. A., Abaci, S., y Morrone, A. S. (2016). Learning technologies badges for faculty professional development: A case study. En Digital Badges in Education: Trends, Issues, and Cases (pp. 249-260). https://doi.org/10.4324/9781315718569

Halavais, A. M. C. (2012). A Genealogy of Badges. Information, Communication and Society, 15(3), 354-373. https:// doi.org/10.1080/1369118X.2011.641992

Hamari, J. (2015). Do badges increase user activity? A field experiment on the effects of gamification. Computers in Human Behavior, 71, 469-478. https:// doi.org/10.1016/j.chb.2015.03.036

Hennah, N., \& Seery, M. K. (2017). Using Digital Badges for Developing High School Chemistry Laboratory Skills. Journal of chemical education, 94(7), 844848. https://doi.org/10.1021/acs.jchemed.7b00175

Hickey, D. T., Otto, N., Itow, R., Schenke, K., Tran, C., y Chow, C. (January 2014). Badges Design Principles Documentation Project Interim Report. Center for Research on Learning and Technology. Indiana
University.

Hickey, D. T., y Chartrand, G. T. (2020). Recognizing competencies vs. completion vs. participation: Ideal roles for web-enabled digital badges. Education and Information Technologies, 25(2), 943-956. https://doi.org/10.1007/s10639-01910000-w

Hunsaker, E., y West, R. E. (2020). Designing Computational Thinking and Coding Badges for Early Childhood Educators. TechTrends, 64(1), 7-16. https://doi. org/10.1007/s11528-019-00420-3

Hurst, E. J. (2015). Digital Badges: Beyond Learning Incentives. Journal of Electronic Resources in Medical Libraries, 12(3), 182-189. https://doi.org/10 $.1080 / 15424065.2015 .1065661$

Jones, W. M., Monty Jones, W., Hope, S., y Adams, B. (2018). Teachers' perceptions of digital badges as recognition of professional development. En British Journal of Educational Technology (Vol. 49, Issue 3, pp. 427-438). https://doi.org/10.1111/bjet.12557

Kappes, S., y Betro, V. C. (2015). Using Mozilla badges to certify XSEDE users and promote training. Proceedings of the 2015 XSEDE Conference: Scientific Advancements Enabled by Enhanced Cyberinfrastructure, 1-4. https://doi. org/10.1145/2792745.2792759

Keane, J., Otter, M., Oxley, T., y Lipscomb, L. (2016). VIF international education: Global-ready teacher badging. En L. Y. Muilenburg y Z. L. Berge (Eds.), Digital Badges in Education: Trends, Issues and Cases (pp. 226-237). Routledge, New York.

Kullaslahti, J., Ruhalahti, S., y Brauer, S. (2019). Professional Development of Digital Competences: Standardised Frameworks Supporting Evolving Digital Badging Practices. En Journal of Siberian Federal University. Humanities y Social Sciences (Vol. 12, Issue 2, pp. 175-186). https://doi.org/10.17516/1997-1370-0387

Liberati, A., Altman, D. G., Tetzlaff, J., Mulrow, C., Gøtzsche, P. C., Ioannidis, J. P. A., Clarke, M., Devereaux, P. J., Kleijnen, J., y Moher, D. (2009). The PRISMA statement for reporting systematic reviews and meta-analyses of studies that evaluate health care interventions: explanation and elaboration. Journal of Clinical Epidemiology, 62(10), e1-e34. https://doi.org/10.1016/j.jclinepi.2009.06.006

Lius, E. (2016). Gamify and Recognize Prior Learning: How to Succeed in Educators' Further Professional Training with Open Badges. International Association for Development of the Information 
Society (IADIS) International Conference on e-Learning, Madeira, Portugal.

Livneh, C., y Livneh, H. (1999). Continuing Professional Education among Educators: Predictors of Participation in Learning Activities. Adult Education Quarterly, 49(2), 91-106. https://doi. org/10.1177/074171369904900202

Liyanagunawardena, T. R., Scalzavara, S., y Williams, S. A. (2017). Open badges: A systematic review of peer-reviewed published literature (2011-2015). European Journal of Open, Distance and E-learning, 2O(2), 1-16. https://doi.org/10.1515/eurodl-2017-0013 Lockwood, C., Dos Santos, K. B., y Pap, R. (2019). Practical Guidance for Knowledge Synthesis: Scoping Review Methods. Asian Nursing Research, 13(5), 287-294. https://doi.org/10.1016/j.anr.2019.11.002

Madero Gómez, S. M. (2010). Factores relevantes del desarrollo profesional y de compensaciones en la carrera laboral del trabajador. Contaduría y Administración, 232, 109-130. https://doi. org/10.22201/fca.24488410e.2010.236

Mah, D.-K., e Ifenthaler, D. (2018). Students' perceptions toward academic competencies: The case of German first-year students. Issues in Educational Research, 28(1), 120-137.

Marr, B. (2020, abril 17). 8 Job Skills To Succeed In A Post-Coronavirus World. Forbes. https://www. forbes.com/sites/bernardmarr/2020/04/17/8job-skills-to-succeed-in-a-post-coronavirusworld/\#674735f22096

Morris, B. J., Dragovich, C., Todaro, R., Balci, S., \& Dalton, E. (2019). Comparing badges and learning goals in low- and high-stakes learning contexts. Journal of Computing in Higher Education, 31(3), 573-603. https://doi.org/10.1007/s12528-019-09228-9

Motheeram, P., Herselman, M., y Botha, A. (2018). A scoping review of digital open badge ecosystems in relation to resource-constrained environments. The Journal for Transdisciplinary Research in Southern Africa. https://doi.org/10.4102/td.v14i1.463

Munn, Z., Peters, M. D. J., Stern, C., Tufanaru, C., McArthur, A., y Aromataris, E. (2018). Systematic review or scoping review? Guidance for authors when choosing between a systematic or scoping review approach. BMC medical research methodology, 18(1). https://doi.org/10.1186/s12874-018-0611-x

Ostashewski, N., y Reid, D. (2015). A history and frameworks of digital badges in education. En Gamification in Education and Business (pp. 187-200). https://doi. org/10.1007/978-3-319-10208-5_10

Otto, N., y Hickey, D. T. (2014). Design principles for digital badge systems a comparative method for uncovering lessons in ecosystem design. En Lecture Notes in Computer Science (including subseries Lecture Notes in Artificial Intelligence and Lecture Notes in Bioinformatics) (Vol. 8699, pp. 179-184). https://doi.org/10.1007/978-3-319-13296-9_20

Peterson, J., Pearce, P. F., Ferguson, L. A., y Langford, C. A. (2017). Understanding scoping reviews: Definition, purpose, and process. Journal of the American Association of Nurse Practitioners, 29(1), 12-16. https://doi.org/10.1002/2327-6924.12380

Pham, M. T., Rajić, A., Greig, J. D., Sargeant, J. M., Papadopoulos, A., y McEwen, S. A. (2014). A scoping review of scoping reviews: advancing the approach and enhancing the consistency. Research Synthesis Methods, 5(4), 371-385. https://doi.org/10.1002/ jrsm.1123

Pineda, P., y Sarramona, J. (2006). El nuevo modelo de formación continua en España: balance de un año de cambios. Revista de Educación, 341, 705-736.

Posgrados y Educación Continua. (2017, mayo 29). Insignias de Educación Continua del Tecnológico de Monterrey. https://www.youtube.com/watch?v= uBKWzqUpfO

PRISMA Flow Diagram. (2009). Recuperado el 18 de noviembre de 2019 a partir de http://prismastatement.org/PRISMAStatement/FlowDiagram. aspx

Raish, V., y Rimland, E. (2016). Employer perceptions of critical information literacy skills and digital badges. College and Research Libraries, 77(1), 87113. https://doi.org/10.5860/crl.77.1.87

Reynoso Flores, M., Castillo Elizondo, J. A., Rangel, D., y Isabel, M. (2014). La formación integral del estudiantado de ingeniería a través de la educación continua. Revista Electrónica Educare, 18(1), 77-96.

Roy, S., y Clark, D. (2019). Digital badges, do they live up to the hype? British Journal of Educational Technology, 5O(5), 2619-2636. https://doi.org/10.1111/bjet.12709

Schwarz, S. J. (2016). Digital badge adoption: earner's perceived educational value (A.-P. Correia (Ed.)) [Thesis. Master of Science]. Iowa State University.

Shields, R., y Chugh, R. (2017). Digital badges - rewards for learning? Education and Information Technologies, 1-8. https://doi.org/10.1007/s10639-016-9521-x

Taylor, J. M., \& Neimeyer, G. J. (2017). The ongoing evolution of continuing education. En T. Rousmaniere, 
Goodyear, Rodney K., Miller, Scott D., y B. E. Wampold (Eds.), The Cycle of Excellence: Using Deliberate Practice to Improve Supervision and Training (pp. 219-248). Wiley Online Library. https://doi.org/10.1002/9781119165590.ch11

Tricco, A. C., Lillie, E., Zarin, W., O’Brien, K. K., Colquhoun, H., Levac, D., Moher, D., Peters, M. D. J., Horsley,
T., Weeks, L., Hempel, S., Akl, E. A., Chang, C., McGowan, J., Stewart, L., Hartling, L., Aldcroft, A., Wilson, M. G., Garritty, C., ... Straus, S. E. (2018). PRISMA Extension for Scoping Reviews (PRISMASCR): Checklist and Explanation. Annals of Internal Medicine, 169(7), 467-473. https://doi.org/10.7326/ M18-0850

RIDU / Revista Digital de Investigación en Docencia Universitaria / e-ISSN: 2223-2516

(C) Los autores. Este artículo es publicado por la Revista Digital de Investigación en Docencia Universitaria del Área de Institutional Research and Effectiveness de la Dirección de Aseguramiento de la Calidad, Universidad Peruana de Ciencias Aplicadas. Este es un artículo de acceso abierto, distribuido bajo los términos de la LicenciaCreativeCommons Atribución-CompartirIgual 4.0 Internacional.( http://creativecommons.org/licenses/by-sa/4.0/), que permite el uso no comercial, distribución y reproducción en cualquier medio, siempre que la obra original sea debidamente citada. 